Invited paper

\title{
Infection prevention and control in Sri Lankan hospitals in relation to WHO Guidelines
}

\author{
Kushlani Jayatilleke ${ }^{1}$ \\ Sri Lankan Journal of Infectious Diseases 2017 Vol.7 (1):2-9 \\ DOI: http://doi.org/10.4038/sljid.v7i1.8131
}

\begin{abstract}
Healthcare associated Infections (HAI or HCAI) are a major problem in healthcare settings. Healthcare associated infections are defined as infections which occur in a patient during the process of care in a hospital or any other healthcare facility which was not present or incubating at the time of admission. Properly conducted Infection Prevention and Control (IPC) programmes are essential to reduce the burden of HCAIs as well as antimicrobial resistance (AMR). Guidelines on Core Components of Infection Prevention and Control Programmes at the National and Acute Health Care Facility Level according to World Health Organisation (WHO) recommends 8 core components. In this article IPC practices in Sri Lanka are examined in comparison to the WHO guidelines.
\end{abstract}

Keywords: IPC, Sri Lanka, Infection Prevention and Control, WHO IPC guidelines

\section{Introduction}

Healthcare Associated Infections (HAI or HCAI) are a major problem in any healthcare setting. HCAIs are defined as infections which occur in a patient during the process of care in a hospital or any other healthcare facility, which was not present or incubating at the time of admission. ${ }^{1}$ These infections can appear even after discharge from hospital. They also include occupational infections among staff. HCAI represents the most frequent adverse event during care delivery and no institution or country can claim to have solved the problem yet. Based on data from a number of countries, it is estimated that each year, hundreds of millions of patients around the world are affected by HCAI. The burden of HCAI is several folds higher in low and middle income countries than in high income ones. ${ }^{1}$ Every day, HCAI results in prolonged hospital stays, long-term disability, increased resistance of microorganisms to antimicrobials, massive additional costs for health systems, high costs for patients and their families, and unnecessary deaths. ${ }^{1}$ Properly conducted IPC programmes are essential to reduce the burden of HCAIs as well as antimicrobial resistance (AMR).

World Health Organisation (WHO) guidelines on core components of IPC programmes at the national and acute healthcare facility level provide 8 core components as given in Table $1 .{ }^{1}$

${ }^{1}$ Sri Jayawardenapura Hospital, Nugegoda, Sri Lanka

Address for correspondence: 393/c, 3rd Lane, Dutugemunu Mawatha, Battaramulla, Sri Lanka. Tel: +94714145056 Email: kush_jaya@yahoo.co.uk

Received 15 February 2017 and accepted 9 March 2017 
Table 1: Core components of IPC programmes (WHO) ${ }^{1}$

\begin{tabular}{|c|c|c|}
\hline $\begin{array}{c}\text { Core component } \\
1\end{array}$ & Infection prevention and control programmes & $\begin{array}{l}\text { 1a Healthcare facility level } \\
\text { 1b National level }\end{array}$ \\
\hline $\begin{array}{c}\text { Core component } \\
2\end{array}$ & $\begin{array}{l}\text { National and facility level infection prevention } \\
\text { and control guidelines }\end{array}$ & $\begin{array}{l}2 \text { a National level } \\
2 \text { b Healthcare facility level }\end{array}$ \\
\hline $\begin{array}{c}\text { Core component } \\
3 \\
\end{array}$ & $\begin{array}{l}\text { Infection prevention and control education and } \\
\text { training }\end{array}$ & $\begin{array}{l}\text { 3a Healthcare facility level } \\
\text { 3b National level }\end{array}$ \\
\hline $\begin{array}{l}\text { Core component } \\
4 \\
\end{array}$ & Healthcare-associated infection surveillance & $\begin{array}{l}\text { 4a Healthcare facility level } \\
\text { 4b National level }\end{array}$ \\
\hline $\begin{array}{l}\text { Core component } \\
5\end{array}$ & $\begin{array}{l}\text { Multimodal strategies for implementing } \\
\text { infection prevention and control activities }\end{array}$ & $\begin{array}{l}\text { 5a Healthcare facility level } \\
\text { 5b National level }\end{array}$ \\
\hline $\begin{array}{l}\text { Core component } \\
6\end{array}$ & Monitoring and evaluation and feedback & $\begin{array}{l}\text { 6a Healthcare facility level } \\
\text { 6b National level }\end{array}$ \\
\hline $\begin{array}{c}\text { Core component } \\
7 \\
\end{array}$ & $\begin{array}{l}\text { Workload, staffing and bed occupancy at the } \\
\text { facility level }\end{array}$ & \\
\hline $\begin{array}{l}\text { Core component } \\
8\end{array}$ & $\begin{array}{l}\text { Built environment, materials and equipment for } \\
\text { infection prevention and control at the facility } \\
\text { level }\end{array}$ & $\begin{array}{l}\text { 8a General principles } \\
\text { 8b.Materials, equipment and } \\
\text { ergonomics for appropriate hand } \\
\text { hygiene }\end{array}$ \\
\hline
\end{tabular}

\section{Core component 1: Infection prevention and control programmes}

\section{1a Healthcare facility level}

According to the WHO guidelines, an IPC programme with a dedicated, trained team should be in place in each acute healthcare facility for the purpose of preventing HAI and combating AMR through IPC good practices. The guidelines also state that a minimum ratio of one full-time or equivalent infection preventionist (nurse or doctor) per 250 beds should be available. However, there was a strong opinion that a higher ratio should be considered, for example, one infection preventionist per 100 beds, due to increasing patient acuity and complexity, as well as the multiple roles and responsibilities of the modern infection preventionist.

\section{In Sri Lanka:}

In Sri Lanka most of the major hospitals have an IPC programme with a dedicated team with trained nurses. The training of nurses on IPC is carried out in Colombo annually by the Ministry of Health. However, in most Sri Lankan hospitals, not even the minimum ratio of one infection preventionist per 250 beds is maintained. IPC units in hospitals are headed by the Consultant Microbiologists, when they are available. All major (Provincial and Teaching) hospitals of the country have full time consultant microbiologists trained locally and overseas (United Kingdom or Australia) and Board Certified as Specialists in Medical Microbiology by the Board of Study in Medical Microbiology of the Post Graduate Institute of Medicine, University of Colombo, Sri Lanka. Training (including the overseas component) is state funded.

Good quality microbiological laboratory support is a very critical factor for an effective IPC programme. ${ }^{1}$ A key drawback in Sri Lanka is the inadequacy of microbiologists as all hospitals do not have the required specialist services and with the exception of the National Hospital of Sri Lanka, each hospital has only one Consultant Microbiologist though the bed strength of several are greater than $1000 .^{2}$ Only a few IPC units have medical officers who are trained in 
IPC. Nurses specially trained on IPC are available in most hospitals. Nurses who have experience after basic training are specially trained in IPC initially for 2 weeks and then periodically by the Ministry of Health of Sri Lanka. Liaison or link nurses who have IPC training are available in each unit or ward in some hospitals.

Currently, none of the microbiology laboratories in state hospitals have accreditation with the Sri Lanka Accreditation Board, though some are working towards accreditation.

\section{1b National level}

It is recommended that active, stand alone, national IPC programmes with clearly defined objectives, functions and activities should be established for the purpose of preventing HAI and combating AMR through IPC good practices. ${ }^{1}$ National IPC programmes should be linked with other relevant national programmes and professional organizations.

The guidelines also state that the minimum objectives of a national IPC programme should include goals to be achieved for endemic and epidemic infections and development of recommendations for IPC processes and practices that are known to be effective in preventing HCAI and the spread of AMR.

The International Health Regulations (IHR: 2005) ${ }^{3}$ and the WHO Global Action Plan on AMR $(2015)^{4}$ support national level action on IPC as a central part of health systems' capacity building and preparedness. This includes the development of national plans for preventing HAI, the development or strengthening of national policies and standards of practice regarding IPC activities in health facilities, and the associated monitoring of the implementation of and adherence to these national policies and standards. The guideline further recommends that the organization of the programme should include (but not be limited to) at least the following components as shown in Table 2.

\section{Table 2: Minimum components for inclusion in IPC}

1. Appointment of technical team of trained infection preventionists (medical/nursing professionals)

2. Formal IPC training of the technical teams and allocated time according to tasks

3. The team should have the authority to make decisions and to influence field implementation and be provided with a protected and dedicated budget according to planned IPC activity and supported by national authorities and leaders

4. Establishment and maintenance of linkages between the national IPC programme and other related programmes

5. Establishment of an official multidisciplinary group, committee or an equivalent structure to interact with the IPC technical team

\section{In Sri Lanka:}

In Sri Lanka there is no national IPC programme or a technical team to carry out IPC work at national level.

The National Health Strategic Master Plan 2016 - 2025 (Curative Services) Ministry of Health of Sri Lanka has identified IPC in healthcare settings and combating antimicrobial resistance as two national programmes to be implemented during the period 2016-2025. Outcome indicators and a monitoring and evaluation plan have been identified in both these programmes.

Three national indicators related to IPC have been identified in consultation with the Sri Lanka College of Microbiologists. These 3 indicators, Staphylococcus aureus bacteraemia rates, 
surgical site infection (SSI) rates following Lower Segment Caesarean Section (LSCS), and hand hygiene compliance rates are currently being monitored by the healthcare quality and safety directorate of the Ministry of Health. S. aureus bacteraemia rate per 10,000 patient- days, MRSA bacteraemia rate per 10,000 patient-days, and hospital onset MRSA bacteraemia rate per 10,000 patient-days and proportion of MRSA: S. aureus in blood cultures (expressed as a \% ) are being monitored in hospitals with facilities for blood culture.

\section{Core component 2: National and facility level infection prevention and control guidelines}

The WHO guidelines recommend that evidence-based guidelines be developed and implemented for the purpose of reducing HAI and AMR. The education and training of relevant healthcare workers on the guideline recommendations and the monitoring of adherence with guideline recommendations should be undertaken to achieve successful implementation.

\section{2a National level}

Development of evidence-based guidelines and their implementation strategies is a key function of the national IPC programme. The national IPC programme should ensure that the necessary resources such as infrastructure and supplies are available and it should support and mandate healthcare worker education and training based on these guidelines.

\section{In Sri Lanka:}

In Sri Lanka, evidence-based National IPC Guidelines are not available. An Infection Control Manual $^{5}$ was written based on available evidence and compiled and published by the Sri Lanka College of Microbiologists (SLCM) and is used by most healthcare institutions in the country and is currently being revised.

\section{2b. Healthcare facility level}

Appropriate IPC expertise is necessary to write or adapt and adopt a guideline, both at the national and healthcare facility level. ${ }^{1}$ Guidelines should be evidence-based and reference international or national standards. Adaptation to local conditions should be considered for the most effective uptake and implementation. Monitoring adherence to guideline implementation is essential.

\section{In Sri Lanka:}

Consultant Microbiologists have developed local IPC guidelines at facility level based on international guidelines and the Infection Control Manual of the Sri Lanka College of Microbiologists. Audits are carried out to monitor the adherence to guidelines implementation.

\section{Core component 3: Infection prevention and control education and training}

\section{3a Healthcare facility level}

The WHO guidelines recommend that IPC education should be in place for all healthcare workers by utilizing team and task-based strategies that are participatory and include bedside and simulation training, to reduce the risk of HAI and AMR. IPC education and training should be a part of an overall health facility education strategy, including new employee orientation and the provision of continuous educational opportunities for existing staff, regardless of level and position (for example, including also senior administrative and housekeeping staff). Three categories of human resources were identified as targets for IPC training and requiring different strategies and training contents: IPC specialists, all healthcare workers involved in service delivery and patient care, and other personnel that support health service delivery 
(administrative and managerial staff, auxiliary service staff, cleaners, etc.). Periodic evaluations of both the effectiveness of training programmes and assessment of staff knowledge should be undertaken on a routine basis.

\section{In Sri Lanka:}

In Sri Lanka some individual healthcare facilities carry out education and training based on a pre-planned programme. Evaluation of the effectiveness of the training programme is not performed in most of the centres and assessment of the staff knowledge is done inconsistently.

\section{3b National level}

The national IPC programme should support the education and training of the health workforce as one of its core functions. ${ }^{1}$

\section{In Sri Lanka:}

In Sri Lanka though there is no national IPC programme, training of IPC staff, specially IPC nurses, are carried out annually by the Ministry of Health along with the Consultant Microbiologists. A training programme has been commenced by the Ministry of Health along with SLCM, for medical officers in IPC units and hopes to continue it annually.

\section{Core component 4: Healthcare-associated infection surveillance}

\section{4a. Healthcare facility level}

The guideline recommends that facility-based HAI surveillance should be performed to guide IPC interventions and detect outbreaks, including AMR surveillance with timely feedback of results to healthcare workers and stakeholders through national networks.

\section{In Sri Lanka:}

Surveillance of HAI and AMR is carried out only in a few Sri Lankan hospitals and feedback is given to the relevant hospital administrators and the consultants. S. aureus bacteraemia rates and SSI rates following LSCS are monitored through the Healthcare Quality and Safety Directorate of the Ministry of Health as previously stated.

\section{4b. National level}

The guidelines recommend that national HAI surveillance programmes and networks that include mechanisms for timely data feedback and with the potential to be used for benchmarking purposes should be established to reduce HAI and AMR.

\section{In Sri Lanka:}

S. aureus bacteraemia rates and surgical site infection rates following LSCS are monitored through the Healthcare Quality and Safety Directorate of the Ministry of Health but a proper system for timely feedback and use for benchmarking is not yet established.

\section{Core component 5: Multimodal strategies for implementing infection prevention and control activities}

\section{5a. Healthcare facility level}

The guideline recommends that IPC activities using multimodal strategies should be implemented to improve practices and reduce HAI and AMR. 
A multimodal strategy consists of several of elements or components (3 or more; usually 5) implemented in an integrated way with the aim of improving an outcome and changing behaviour. It includes tools, such as bundles and checklists developed by multidisciplinary teams that take into account local conditions. The 5 most common components to be included are shown in Table 3.

Table 3: Components most commonly included in a multimodal strategy for IPC

1. system change (availability of appropriate infrastructure/supplies to enable IPC good practices)

2. education and training of healthcare workers/key players (for example, managers)

3. monitoring infrastructures/practices/processes/outcomes and providing data feedback

4. reminders in the workplace/communications

5. culture change with the establishment and strengthening of a safety climate

\section{In Sri Lanka:}

In Sri Lankan healthcare facilities where Consultant Microbiologists and trained IPC staff are available, multimodal strategies are implemented.

\section{5b. National level}

The guidelines recommend that national IPC programmes should coordinate and facilitate the implementation of IPC activities through multimodal strategies on a nationwide or sub-national level.

\section{In Sri Lanka:}

In Sri Lanka there is no national programme through which implementation of multimodal strategies are monitored and facilitated.

\section{Core component 6: Monitoring/audit of IPC practices and feedback and control activities}

\section{6a. Healthcare facility level}

The guideline recommends that regular monitoring/audit and timely feedback of healthcare practices according to IPC standards should be performed to prevent and control HAI and AMR at the healthcare facility level. Feedback should be provided to all audited persons and relevant staff.

\section{In Sri Lanka:}

Hand hygiene audits are performed in most of the major Sri Lankan hospitals though the consistency is not monitored. Feedback is given to relevant staff during the audit as well as later at Infection Control Committee (ICC) meetings and in report format. Audits on other practices are done only in a few facilities.

\section{6b. National level}

The panel recommends that a national IPC monitoring and evaluation programme should be established to assess the extent to which standards are being met and activities are being performed according to the programme's goals and objectives. Hand hygiene monitoring with feedback should be considered as a key performance indicator at the national level.

In Sri Lanka: Hand hygiene compliance rate is identified as a national indicator by the Ministry of Health of Sri Lanka. Available data (personal communication) show that the average hand 
hygiene compliance in 2015 was $60 \%$. Currently the feedback is not given to the relevant healthcare setting for bench marking.

\section{Core component 7: Workload, staffing and bed occupancy at the facility level}

The panel recommends that the following elements should be adhered to in order to reduce the risk of HAI and the spread of AMR: Firstly, bed occupancy should not exceed the standard capacity of the facility and secondly, healthcare worker staffing levels should be adequately assigned according to patient workload.

\section{In Sri Lanka:}

In most of the public sector hospitals the bed occupancy exceeds $100 \%$ thus making it essential for patients to share the beds. This violates fundamental principles of IPC. Healthcare worker staffing levels too are grossly inadequate. The total number of nurses and doctors in public hospitals were 32,362 and 17,974 (1,573 specialists and 16,401 grade medical officers) by end of 2015. ${ }^{7}$ This is for a population of 20.8 million (20,810,816 in 2016 and 20,715,010 in 2015). In most instances the ideal nurse to patient ratio of 1:1 in Intensive Care Unit (ICU) settings is not maintained.

\section{Core component 8: Built environment, materials and equipment for IPC at the facility level}

\section{8a General principles}

Patient care activities should be undertaken in a clean and/or hygienic environment that facilitates practices related to the prevention and control of HAI, as well as AMR, including all elements around water, sanitation and hygiene (WASH) infrastructure and services and the availability of appropriate IPC materials and equipment.

\section{In Sri Lanka:}

Disposable gloves and other personal protective equipment (PPE) are available in most healthcare facilities but uninterrupted supply of IPC material and equipment is not guaranteed throughout the year for most public sector hospitals. Consultant Microbiologists have a monthly meeting at the Ministry of Health with the Director General of Health Services (DGHS) and deficiencies are informed to the DGHS and solutions obtained. The use of disposable needles and syringes for blood collection for laboratory investigations is now implemented.

\section{8b Materials, equipment and ergonomics for appropriate hand hygiene}

The panel recommends that materials and equipment to perform appropriate hand hygiene should be readily available at the point of care; i.e. The place where 3 elements come together: the patient, the healthcare worker and care or treatment involving contact with the patient or his/her surroundings (within the patient zone). The concept embraces the need to perform hand hygiene at recommended moments exactly where care delivery takes place. This requires that a hand hygiene product (for example, alcohol-based hand rub, if available) be easily accessible and as close as possible - within arm's reach of where patient care or treatment is taking place. Point-of-care products should be accessible without having to leave the patient zone. The WHO Guidelines on hand hygiene in healthcare state: "minimum sink-to-bed ratio 1:10 and 1:1 in isolation rooms." 4 


\section{In Sri Lanka:}

Alcohol based hand rub is available in most major hospitals in Sri Lanka. In most hospitals this is prepared in-house but the acceptability of this product among users is not good as a result of which a decision was taken to buy commercially available alcohol based hand rub for ICUs. In some ICUs the hand rub is available at each bed. Continuous uninterrupted supply of hand disinfecting solutions is still not assured in most healthcare centres in the country (personal observation).

\section{References:}

1. Guidelines on Core Components of Infection Prevention and Control Programmes at the National and Acute Health Care Facility Level; World Health Organization 2016 ISBN 978-92-4-154992-9

2. http://203.94.76.60/nihs/BEDS/Beds2010/Beds2010.pdf downloaded 7.3.17

3. International Health Regulations, third edition. Geneva: World Health Organization; 2005 http://www.who.int/ihr/publications/9789241580496/en/, accessed 18 October 2016).

4. Global action plan on antimicrobial resistance. Geneva: World Health Organization; 2015 http://www.wpro.who.int/entity/drug_resistance/ resources/global_action_plan_eng.pdf, accessed 18 October 2016

5. Hospital Infection Control Manual, First Edition 2005, ISBN 955-8891-01-0; Sri Lanka College of Microbiologists. Accessible at www.slmicrobiology.org

6. WHO Guidelines on Hand Hygiene in Health Care; First global patient safety challenge clean care is safer care; World Health Organization 2009 www.who.int/gpsc/5may/tools/9789241597906/en/

7. Human Resource Profile Ministry of Health; Health Staff in position by 31.12.2015 http://www.health.gov.lk/moh_final/english/public/elfinder/files/publications/HRMprofile2016.pd $f$ 